\title{
Lidil
}

Revue de linguistique et de didactique des langues

$35 \mid 2007$

Figures de l'auteur en didactique

\section{L'énonciation dans les manuels scolaires de troisième}

Françoise Boch et Francis Grossmann

\section{(2) OpenEdition}

Journals

Édition électronique

URL : http://journals.openedition.org/lidil/2163

DOI : 10.4000/lidil.2163

ISSN : $1960-6052$

Éditeur

UGA Éditions/Université Grenoble Alpes

Édition imprimée

Date de publication : 1 juin 2007

Pagination : 25-40

ISBN : $978-2-84310-100-7$

ISSN : $1146-6480$

Référence électronique

Françoise Boch et Francis Grossmann, «L'énonciation dans les manuels scolaires de troisième », Lidil [En ligne], 35 | 2007, mis en ligne le 01 décembre 2008, consulté le 01 mai 2019. URL : http:// journals.openedition.org/lidil/2163; DOI : 10.4000/lidil.2163

Ce document a été généré automatiquement le 1 mai 2019

(C) Lidil 


\title{
L'énonciation dans les manuels scolaires de troisième
}

\author{
Françoise Boch et Francis Grossmann
}

Toute démarche didactique passe par une définition de la matière qui est son objet. Michel Dabène (1987 : 17)

1 Objet didactique récemment introduit dans les programmes du collège, domaine de recherche à peine plus ancien et encore peu stabilisé dans le champ de la linguistique, l'énonciation, d'un point de vue théorique, ne se laisse pas facilement appréhender, a fortiori quand il s'agit de la didactiser. En cela, elle représente un exemple particulièrement illustratif de la difficulté pour le concepteur de manuels (et par là-même pour le lecteur, qu'il soit enseignant ou élève) d'opérer un tri dans les contenus - au-delà des entrées fournies par les Instructions officielles (Io) - et de les mettre en cohérence de façon à donner un sens à leur apprentissage. Délicat en effet, pour ne pas dire impossible, de traiter didactiquement la linguistique énonciative dans sa globalité, tant ce champ aux frontières mouvantes recouvre de notions complexes ayant partie liée avec d'autres domaines eux-mêmes très vastes (temporalité, modalisation, argumentation, point de vue, etc.).

2 Plutôt que de mener une simple étude descriptive des manuels visant à renforcer négativement ces constats ${ }^{1}$, nous avons pris le parti d'orienter nos observations sous un angle particulier : rendre un peu plus cohérente l'approche didactique de la linguistique énonciative requiert selon nous avant tout de problématiser la notion autour d'un axe fédérateur, si tant est que cela soit possible. Tel est en tout cas l'enjeu de cette contribution: comment définir, au plan didactique, une «porte d'entrée " à la problématique de l'énonciation, dont on sait qu'elle est caractérisée par une certaine hétérogénéité terminologique et définitoire, même si l'étiquette "énonciation » se veut fédératrice et homogénéisante ${ }^{2}$ ?

3 L'analyse s'appuie sur l'observation d'un corpus de 9 manuels de français de 3e publiés en $2003^{3}$. Pour chacun des manuels, nous avons relevé les différentes façons de théoriser l'énonciation à travers les définitions qui en étaient données. Ce premier tour d'horizon, 
nous a permis également de cerner les traces (ou l'absence de traces) de mise en correspondance des notions, en particulier celles de "situation d'énonciation", «modalisation » et « discours rapporté », notions qui traversent l'ensemble des manuels et qui figurent explicitement dans les Io. Le traitement didactique des deux notions de modalisation et de discours a fait ensuite l'objet d'une analyse critique visant à mettre au jour ce qui nuit à la cohérence dans leur présentation aux élèves.

\section{Une approche de l'énonciation par la deixis ${ }^{4}$}

Un premier constat s'impose à l'analyse globale des manuels : du fait sans doute de sa complexité théorique et de son caractère trop englobant déjà soulignés, l'énonciation ne fait généralement pas l'objet d'une présentation en tant que telle. Elle est parfois définie de manière assez abstraite en quelques mots dans les aide-mémoires ou glossaires: « l'énonciation, c'est la mise en œuvre du langage, le fait de réaliser

un énoncé » (NAT: 325). «Enonciation : production d'un énoncé » (BEL: 326). Le plus souvent cependant, la notion apparait dans le chapitre consacré à la situation d'énonciation ${ }^{5}$, elle-même décrite, conformément aux Io, à partir d'une opposition binaire très réductrice : énoncé ancré vs énoncé coupé, à laquelle on intègre en classe de 3e l'énoncé mixte (combinant les deux systèmes), trilogie travaillée à partir du repérage des marques supposées caractériser chaque plan énonciatif. L'entrée dans l'énonciation se fait donc le plus souvent par la deixis; or, la complexité du système énonciatif interdit toute répartition exclusive d'un système de marques stables pour catégoriser un énoncé « ancré » ou « coupé » de la situation d'énonciation. De ce fait, ces activités de repérage paraissent insatisfaisantes ${ }^{6}$.

Notre deuxième constat est conforme à notre intuition : les notions de modalisation et de discours rapporté ne sont que très allusivement (quand elles le sont) mises en perspective avec la notion d'énonciation. Pour le discours rapporté, le lien passe le plus souvent par le métalangage : les pronoms de première et deuxième personnes (évoqués dans le passage traitant des "paroles rapportées») sont par exemple désignés en tant qu' indices d'énonciation ${ }^{7}$ » (NAT; BOR). L'opposition ancré/coupé est également mentionnée dans le chapitre sur le discours rapporté, à propos des marques de personne, de lieu et de temps ( $\mathrm{HACH}: 22)$ ou du discours direct (HACH-Text : 370). Quant à la notion de modalisation, les relations qu'elle entretient avec l'énonciation sont encore plus incertaines ${ }^{8}$, même si certains sommaires la font figurer, avec d'autres notions, sous un même chapeau intitulé «l'énoncé » (HAT) ou «Discours et énonciation » (BOR). Force est de constater que ces regroupements (d'ailleurs hétérogènes suivant les manuels) ne sont que de façade; en tout état de cause, ils ne donnent pas lieu à une présentation unifiante du domaine justifiant ces choix.

\section{Modalisation - le couple piège de l'objectivité/ subjectivité}

6 L'approche de la modalisation dans les manuels met au jour la difficulté à cerner la notion et à en proposer une définition claire et utile pour les élèves. M.-L. Elalouf et M. Kerinec (1999) avaient déjà bien identifié en quoi elle pose problème dans le cadre de l'enseignement ; la variabilité à l'œuvre dans la définition qu'en donnent les ouvrages de 
référence serait l'une des raisons du caractère insaisissable de la notion, plus ou moins extensible. Si on l'entend classiquement, et c'est le cas dans les manuels, comme le processus par lequel le sujet de l'énonciation manifeste son attitude à l'égard de son discours, alors la modalisation - synonyme de subjectivité ${ }^{9}$ - est partout : « la présence de l'énonciateur est consubstantielle à l'énoncé » (Elalouf et Kerinec, 1999: 100). On ne peut alors s'étonner des listes démesurées, soumises à une forte variabilité dans les manuels des marques de modalisation ${ }^{10}$, auxquelles on va tenter de sensibiliser l'élève. Le problème, bien sûr, est que l'entreprise est sans fin, et que les marques appartiennent à des plans divers. Citons par exemple NAT qui propose l'inventaire suivant: «Pronom personnel, formules particulières (pour moi, d'après les scientifiques, etc.), verbes (croire, estimer, prétendre, etc.), adverbes (probablement, etc.), conditionnel, choix du lexique (termes dévalorisants ou valorisants) niveau de langue, comparaison et métaphore ", ou encore DID, qui envisage de façon très large les marques exprimant l'appréciation subjective, incluant le « jugement esthétique ». Dans l'exemple : «c'est là une île très verte, plate et fertile ", les adjectifs «verte» ou " plate» sont considérés comme des marqueurs de subjectivité. Avec une telle analyse, on peut craindre que les élèves se noient dans ces marques et ne discernent plus celles qui leur seraient réellement utiles en production ${ }^{11}$.

7 Au-delà du problème de marquage de la modalisation, la notion même de subjectivité, et son supposé corollaire celle d'objectivité, implique une confusion faisant obstacle à la démarche d'appropriation par les élèves des outils de la modalisation, car dans son opposition à « subjectif », un énoncé « objectif » est assimilé à « neutre » (BOR: 276, HACH : 178, BEL : 327). Ainsi, en listant « les marques plus ou moins visibles de la subjectivité de celui qui parle, c'est-à-dire des sentiments ou des jugements de valeur, positifs ou négatifs qu'il remarque", l'un des manuels signale que "lorsque ces marques sont effacées, l'énoncé est neutre, plus objectif » (NAT : 332).

8 Cette assimilation généralisée du concept de neutralité à celui d'objectivité pose problème : la neutralité ne fait sens que lorsque le locuteur/énonciateur ne prend pas position face à plusieurs points de vue antagonistes ${ }^{12}$. Un énoncé «neutre» (si tant est que la catégorie existe) se définit donc par une absence de prise de position du locuteur (ou de point de vue, dans le sens argumentatif du terme) ; un énoncé « objectif » quant à lui peut comporter des traces du point de vue pris en charge par le locuteur. La distinction entre neutralité et objectivité nous parait fondamentale quand on traite de la modalisation, et aisément assimilable par les élèves ${ }^{13}$ : associée à la notion de genre, elle permet de renverser l'approche de la notion, en l'envisageant non plus à partir des marques (approche dont on a évoqué les risques potentiels de noyade dans la profusion et la confusion des plans d'analyse) mais à partir des opérations énonciatives, dont on fait l'hypothèse qu'elles supportent davantage de sens et sont plus utiles à l'activité d'interprétation ou de production de textes. Il ne s'agit pas de négliger l'analyse des marques, indispensable à l'enrichissement de la palette linguistique des élèves, mais de procéder ainsi à une démarche didactique autorisant une sélection réfléchie de ces outils. Ainsi, dans le cadre du discours argumentatif, lorsque les élèves sont appelés à construire (ou identifier dans un texte) un point de vue, on peut les inviter à puiser dans les outils linguistiques s'inscrivant sur l'axe de la certitude (voir par exemple le tableau exemplifié chez H-EDUC, où l'on répertorie des marques indiquant le degré d'assurance du locuteur). La notion d'effacement énonciatif prend ici tout son sens : il s'agit de défendre un point de vue (et donc, encore une fois, de sortir de la neutralité) tout en centrant le discours sur son objet, et non sur soi. Formulé plus simplement, il s'agit de transmettre l'idée que 
donner son avis sur une question en construisant des arguments n'est pas la même chose qu'exprimer ses sentiments et émotions.

Parallèlement, dans le cadre d'une séquence sur la description, il peut être intéressant de mobiliser la modalité axiologique (allant du favorable au défavorable par exemple, ainsi que le propose $H A T$ ) ou les modalités appréciatives (BEL-G) selon la terminologie employée. Il ne s'agit plus de viser l'objectivation constitutive du discours argumentatif, mais de jouer sur le plan des affects, en les donnant - ou pas, ou plus ou moins - à voir. Dans ce cas précis, on comprend mieux comment «neutre» et "objectif» peuvent s'amalgamer. Ainsi, « objectif » est défini dans un glossaire de manuel comme ce « qui est exact, conforme à la réalité et qui ne reflète en aucun cas le point de vue de celui qui s'exprime. Antonyme de subjectif ». De la même façon, « subjectif » est ainsi caractérisé : «qui reflète les pensées, les sentiments de celui qui parle ou qui écrit. Une description ou un portrait peuvent être objectifs ou subjectifs. Par exemple, un portrait peut refléter la tendresse, l'admiration ou au contraire la haine, le mépris que l'on éprouve pour quelqu'un.»(BOR: 395). La subjectivité est exclusivement reliée aux affects, qui viendraient fausser la réalité « vraie ». Le « point de vue » est assimilé à sentiments, et non à prise de position. On touche sans doute ici à la source de la confusion entre neutralitéet objectivité. Dans la description, les marques de "subjectivité " servent à exprimer le ressenti du locuteur (et leur absence caractériserait un énoncé « neutre » ou « objectif »). Dans l'argumentation en revanche, ces marques dites également de "subjectivité " (pourtant d'une tout autre nature) traduisent au contraire une relation de mise à distance du locuteur avec son objet (et leur absence caractériserait un énoncé en aucun cas neutre, et qui pourrait même être qualifié de " subjectif ${ }^{14}$ »). Dans ce dernier cas où il s'agit de construire un point de vue, ces marques servent (au lieu de s'opposer) à l'objectivité au sens argumentatif du terme.

Afin d'éviter d'embrouiller les élèves avec ces contradictions inhérentes à la terminologie adoptée, il nous semble préférable de ne pas recourir au concept trop englobant de " marques de subjectivité », grand sac fourre-tout qui renvoie à des traces aux fonctions parfois opposées, et de parler plutôt de "traces énonciatives", en distinguant celles servant la modalisation et celles permettant l'expression des affects.

\section{Le discours rapporté : vers une conception polyphonique?}

11 La présentation des «paroles rapportées » (c'est l'expression, un peu réductrice, que l'on retrouve à présent souvent dans les manuels) subit peu de modifications par rapport à la période antérieure. On note cependant quelques acquis indiscutables, comme des tentatives, encore isolées, pour mettre en évidence les différentes fonctions du DR. BEL-G par exemple signale que la citation permet de dégager sa responsabilité pour « donner davantage d'autorité » à une assertion ou bien pour «montrer qu'on n'adhère pas aux propos cités » (BEL-G:101).

Sur la question du statut ontologique du DR, on se trouve dans une situation également incertaine et variable suivant les manuels, une majorité s'en tenant à la vulgate selon laquelle «[le DD] transcrit un dialogue tel qu'il a été prononcé », ce qui permettrait de préserver «l'authenticité du discours » (BOR-TLL: 351). (BEL-G: 102) signale de manière plus juste que les paroles citées sont seulement présentées comme authentiques. 
13 Malgré l'introduction, pertinente, dans certains manuels des pensées rapportées (HAT consacre une leçon aux "paroles et pensées rapportées»), l'intégration du discours rapporté dans une conception plus globale, polyphonique, reste embryonnaire. On ne prend pas en compte les énoncés en "selon $\mathrm{X}$ », « d'après $\mathrm{Z}$ » (« modalisation en discours second» de Authier-Revuz, 1992, 1993), pourtant essentielles pour marquer un point de vue, dans certains genres d'écrits. Bien entendu, il n'est sans doute pas utile, à ce niveau, de proposer un inventaire organisé de toutes les formes de mentions ni d'intégrer le " discours direct libre », comme le suggère encore Authier-Revuz. L'objectif serait plutôt, selon nous, de doter les élèves d'un cadre interprétatif cohérent, qui leur permette de comprendre à quoi correspondent les différentes manières de prendre en compte le discours d'autrui. Or, dans l'ensemble, on ne voit guère cette volonté de poser une grille interprétative. On retrouve ainsi, dans les manuels, posée comme une évidence, la trilogie classique DD/DI/DIL, à quoi s'ajoute désormais, par un emprunt terminologique à Genette (1972 : 189), le « récit de paroles » réduit ici au discours narrativisé. Cette introduction peut sembler une ouverture intéressante, mais son ajout reste peu finalisé. Bordas par exemple ${ }^{15}$ signale dans un encadré («Ce qu'il faut savoir ») : « Il y a quatre manières de rapporter des paroles dans un récit» (BOR-TLL:351).

Moins que la forme doxique, sans doute inévitable dans le genre du manuel scolaire, ce que l'on peut regretter, c'est que ne soient pas davantage caractérisées les finalités principales du discours rapporté ; or celles-ci peuvent se structurer aisément (AuthierRevuz, 1992, 1993) autour de l'opposition montrer vs reformuler, qui ont des implications fortes au plan énonciatif, en termes de prise en charge de l'énoncé. Montrer l'énonciation prêtée à autrui, principalement grâce au discours direct, ou la reformuler, au moyen des différentes sortes de discours indirect, oriente en effet l'usage même qu'on peut faire de ces procédés au sein des différents genres de discours. Mettre l'accent sur cette opposition fondamentale permettrait également de faire le lien avec d'autres notions telles que la modalisation qui rend compte du degré de prise en charge énonciative. Sans cet effort, l'introduction du discours narrativisé représente en définitive un simple ajout aux trois « styles », postulés en dehors de la logique énonciative qui les justifie.

Notons enfin que, de manière générale, la plupart des exercices proposés restent des exercices de transformation d'un « style » à l'autre, essentiellement formels, certes utiles mais peu finalisés. Tout se passe donc comme si l'étude du discours direct au collège avait commencé à intégrer la problématique énonciative, mais que, faute d'un axe clair permettant de lui donner du sens, on restait au milieu du gué.

\section{La notion de prise en charge énonciative, une entrée à privilégier}

16 Si l'on accepte les constats qui découlent de l'analyse des manuels de 3e, on voit à quel point il est difficile de proposer une vision cohérente de l'énonciation. Nous proposons donc en premier lieu de distinguer plus clairement qu'il n'est fait actuellement dans les ouvrages analysés (ainsi que dans les Instructions officielles), entre les deux dimensions fondamentales du système énonciatif, la première concernant ce que l'on pourrait appeler l'espace énonciatif ${ }^{16}$, subsumant la question du repérage référentiel, et la seconde, traitant du sujet modal et de la dimension intersubjective, dont rendrait compte la notion de prise en charge énonciative. Cette dichotomie, si elle laisse non résolu le problème, non 
trivial, de l'articulation des deux notions ${ }^{17}$, permet d'éviter de tout mélanger, et autorise une approche plus raisonnée. Elle permet en particulier de sérier les formes d'aides qui pourraient être apportées aux élèves sur ces deux plans, en référence à des problèmes de lecture ou d'écriture mieux circonscrits. Nous allons nous attacher essentiellement ici à la prise en charge énonciative: la question de l'espace énonciatif, étant donné sa complexité, et les difficultés qu'elle pose, tant pour la théorie énonciative que pour une opérationnalisation didactique, mérite un traitement circonstancié que nous renvoyons à une contribution ultérieure ${ }^{18}$.

17 Il existe en effet une autre possibilité, guère exploitée jusqu'à présent, de mettre en cohérence deux des principales notions clés que l'on trouve généralement traitées isolément dans les manuels, la modalisation et le discours rapporté : c'est de les envisager à partir de la notion de prise en charge énonciative ${ }^{19}$. Cette notion nous parait particulièrement utile, pour aider l'élève aussi bien à analyser des textes, qu'à produire des textes dans lesquels il lui faudra défendre un point de vue personnel en l'appuyant sur des assertions prêtées à d'autres. Il y a en effet un réel enjeu, tant au plan des pratiques de lectures «méthodiques » ou « critiques » qui vont devenir la règle au lycée, qu'au plan des écrits à produire - résumés, commentaires, dissertations - qui vont être de plus en plus demandés dans la suite de la scolarité, à mettre en place les outils nécessaires d'une prise de conscience sur ce plan, l'élève ayant de plus en plus à situer son point de vue par rapport à celui d'autrui ${ }^{20}$.

18 Par ailleurs, cette deuxième direction de travail peut être envisagée de manière relativement autonome par rapport à la première. S'il est juste en effet de remarquer que le locuteur marque, en premier lieu, sa présence à travers les indices de personnes, et qu'il s'« ancre dans le monde » (Bertrand, 1999) à travers la deixis spatiale et temporelle, ce système de marques est différent de celui qu'implique la notion de prise en charge énonciative. Le terme subjectivité se révèle en effet une nouvelle fois trompeur: que les indices de personnes - le fait que le locuteur dise je - soient une "marque du sujet parlant » n'est pas niable. Mais cette subjectivité-là gagne à ne pas être confondue avec celle qui présente l'énonciation comme prise en charge du dire : cette dernière impose toujours, avec les réserves d'usage sur la «non-coïncidence du sujet avec son dire » et sa part d'altérité fondamentale, un certain engagement, voire la construction d'un point de vue, ou sur une autre dimension, l'expression et la mise en scène de ses émotions ou de celle de ces « autres » qu'il fait parler dans son discours.

19 Comment définir alors la notion de prise en charge énonciative? Introduite par Ducrot, puis " reniée » ${ }^{21}$, l'expression a servi dans des cadres théoriques variés: on la retrouve actuellement $\mathrm{Z}$. Guentcheva qui ajoute aux modalités logiques classiques, la manière dont l'énoncé est "médiatisé » (Guentcheva, 1996); les opérations de prise en charge recouvrent alors plusieurs opérations élémentaires: "je dis que ce que je dis est vrai» (assertion), ou « je dis que ce que je dis est possible», ou « je dis que ce que je dis est dit par un autre ou déduit à partir d'indices, ou attesté par un témoin (vu ou entendu)», etc. Cet élargissement nous semble intéressant, notamment dans la perspective d'une intégration du discours rapporté, mais n'a de sens que si l'on accepte de définir un noyau commun: le fait que le locuteur informe sur le statut de la vérité proposé à travers l'énoncé, soit qu'il assume l'assertion, ou signale son degré de probabilité, soit qu'il marque son retrait, et indique qu'il n'en revendique pas totalement la responsabilité (assertion présentée comme émanant d'un tiers, ou inférée à partir d'indices). C'est bien le statut de la vérité de l'énoncé qui est déterminant pour fonder une approche cohérente 
de la prise en charge énonciative ${ }^{22}$, au moins lorsqu'on l'envisage dans son sens le plus spécifique. "Prendre en charge" se décline alors soit à travers l'énonciation d'une assertion, dont on devient alors responsable, soit - et c'est bien un deuxième cas de figure - à travers l'accord que l'on manifeste avec un énoncé, proféré ou écrit par un autre (ou par soi-même à une autre occasion). Inversement, le locuteur peut refuser de prendre en charge un énoncé, soit qu'il manifeste son désaccord, soit qu'il refuse de prendre position et adopte une position de neutralité. Nous rappellerons en outre l'importance de l'ancrage affectif de la prise en charge énonciative, ancrage qui se marque lexicalement à travers différents types de traces. Ce sont ces différentes places de la prise en charge qui nous semblent pouvoir faire l'objet d'un travail didactique, mettant en relation le travail sur la modalisation (qui permet par exemple de préciser le degré de prise en charge) et celui sur le discours rapporté (qui fournit des moyens concrets pour se désengager ou s'abriter derrière l'autorité d'un autre).

Travailler ce concept de prise en charge avec les élèves n'est pas utopique : une telle direction de travail est, par exemple, déjà esquissée par certains programmes de l'enseignement secondaire en Belgique ${ }^{23}$. On y trouve explicitement détaillés, sous la mention "Assurer la prise en charge énonciative", les différents types de marques lexicales permettant d'« inscrire la subjectivité du scripteur ", en traduisant sa réaction émotionnelle ou son évaluation (sous l'axe bon/mauvais et l'axe vrai/faux/incertain); la question des discours rapportés y est traitée sous le même chapeau, à partir de ses fonctions (mise à distance du discours de l'autre, annexion du discours de l'autre). On n'échappe évidemment pas non plus, dans une telle optique, aux simplifications, et cette approche reste trop rudimentaire, dès lors qu'il s'agit, par exemple, d'examiner en finesse les usages les plus complexes du discours indirect libre, dans les textes littéraires - mais cela serait tout aussi vrai des usages journalistiques ou du récit oral. En effet, la question du point de vue, souvent associée à celle de prise en charge, reste traitée ici, sans que soit prise en compte la richesse métaphorique même de la notion de "point de vue », qui implique qu'un lien explicite soit effectué entre le sens perceptif pris en compte par la narratologie et le sens énonciatif (Rabatel, 1998, 2003). Une telle articulation est sans doute possible au niveau du lycée, mais elle semble prématurée pour le niveau que nous avons considéré. Telle que nous l'avons envisagée, la notion de prise en charge énonciative fournit au moins un de ces principes de cohérence que nous cherchions, et représente par là même un progrès par rapport aux présentations faites actuellement dans les manuels.

\section{Pour conclure}

21 Notre analyse des manuels a débouché sur la proposition de distinguer plus clairement deux plans qui nous paraissent devoir faire l'objet d'un traitement spécifique : celui de l'espace énonciatif et celui de la prise en charge énonciative. Nous avons fait le choix, provisoire, de nous limiter au deuxième aspect. La notion de prise en charge énonciative permet en effet, selon nous, de présenter de manière cohérente les aspects liés à la modalisation et au discours rapporté. Elle offre en outre, à travers la notion de sujet modal, la possibilité de traiter à la fois, sans les autonomiser, la question du point de vue, entendu au sens argumentatif du terme, et à celle de l'inscription affective du sujet dans le texte, même si, comme on l'a vu, il peut être intéressant de les distinguer lorsqu'il s'agit d'en repérer les traces linguistiques. Point de vue et responsabilité se trouvent ainsi intégrés à des questions plus traditionnellement traitées dans les classes, comme 
l'analyse du style d'un auteur: s'il n'y a pas lieu de confondre style et énonciation, reconnaissons qu'il existe bien une stylistique de l'énonciation. Un deuxième type de choix, essentiel à nos yeux, consiste à partir des opérations énonciatives vers les traces, plutôt que d'emprunter le chemin inverse, pour éviter de noyer les élèves dans des pratiques de repérage, qui seraient, au sens premier du terme, insignifiantes. Soulignons un dernier point : un élément essentiel et étonnant pour nous dans l'analyse des manuels a été de constater à quel point la notion de subjectivité, surtout lorsqu'elle était opposée à celle d'objectivité, était source de confusion, aussi bien en ce qui concerne la question de la modalisation que celle du discours rapporté. Faire réfléchir les élèves sur la manière dont un énoncé ou un discours est pris en charge à travers des traces énonciatives est un moyen de sortir de telles apories, et par là même de déconstruire des mythes tenaces, encore souvent perpétués dans les manuels. Construire un point de vue d'auteur assurant un discours primaire intégrateur, consiste peut-être avant tout, en didactique du français, à faire ce travail préalable d'explicitation critique, permettant l'opérationnalisation des notions mobilisées.

SITRI, F. \& REBOUL, S. (1998) : L'énonciation dans les manuels scolaires du secondaire, Les Carnets du Cediscor, 5, 93-114.

\section{BIBLIOGRAPHIE}

\section{Références bibliographiques}

AUTHIER-REVUZ, J. (1992) : Repères dans le champ du discours rapporté, L'information grammaticale, $55,38-42$.

AUTHIER-REVUZ, J. (1993) : Repères dans le champ du discours rapporté, L'information grammaticale, $56,10-15$.

BERTRAND, D. (1999) : Discours et énonciation dans les nouveaux programmes de collège, Le Français aujourd'hui, 128, 60-65.

BOUTET, J. (1999) : Pour une activité réflexive sur la langue, Le Français aujourd'hui, 128, 28-39.

DABÈNE, M. (1987) : L'adulte et l'écriture. Contribution à une didactique de l'écrit en langue maternelle, Bruxelles, De Boeck Université.

DENDALE P., COLTIER D. (2005) : La notion de prise en charge ou de responsabilité dans la théorie scandinave de la polyphonie linguistique, in J. Bres, P. P. Haillet, S. Mellet, H. Nolke, Laurence Rosier (éds.) : Dialogisme et polyphonie. Approches linguistique, Bruxelles, De Boeck/Duculot, 125-140.

ELALOUF, M.-L. (1998) : L'énonciation dans les nouveaux sujets du baccalauréat, Les Carnets du Cediscor, 5, 115-133.

ELALOUF, M.-L., KERINEC, M. (1999) : Un groupement de textes sur la modalisation au lycée, Le Français aujourd'hui, 128, 99-109.

GENETTE, G. (1972) : Figures III, Paris, Seuil, Coll. Poétique. 
GUENTCHÉVA Z. (1996) (éd.) : L'Énonciation Médiatisée, Louvain et Paris, Peeters.

KERBRAT-ORECCHIONI, C. (1980) : L'énonciation, de la subjectivité dans le langage, Paris, Armand Colin.

MAINGUENEAU, D. (1993) : Le contexte de l'œuvre littéraire. Enonciation, écrivain, société, Paris, Dunod.

MAINGUENEAU, D. (1999) : Réflexions sur la « grammaire du discours » au collège, Le Français aujourd'hui, 128, 20-28.

MAINGUENEAU, D. (2002) : Article Enonciation, Dictionnaire d'analyse du discours, P. Charaudeau \& D. Maingueneau (éds), Paris, Seuil, 228-231.

MINISTÈRE DE LA COMMUNAUTÉ FRANÇAISE, (2002) : Programme d'études du cours de français, Enseignement professionnel, deuxième et troisième degré, disponible en ligne, (consulté le 18-09-06).

PAVEAU, M.-A. (1999) : Usage et mésusage des théories énonciatives, Le Français aujourd'hui, 128, 110-117.

PUECH, C. (1999) : L'énonciation et les Instructions officielles : remarques sur une réception, Le Français aujourd'hui, 128, 49-59.

RABAtel, A. (1998) : La Construction textuelle du point de vue, Lausanne, Paris, Delachaux et Niestlé.

RABATEL, A. (2003) : Le point de vue, entre langue et discours, description et interprétation : état de l'art et perspectives, Cahiers de praxématique, 41, 7-24.

RABATEL, A. (2004) : La part de l'énonciateur dans la construction interactionnelle des points de vue, Marges linguistiques 9, 115-136.

RINCK, F. (2006) : L'article de recherche en sciences du langage et en lettres : figure de l'auteur et identité disciplinaire du genre, Thèse de doctorat en Sciences du langage, Grenoble 3.

\section{NOTES}

1. On se reportera en particulier aux analyses pertinentes effectuées par Boutet (1999), Elalouf (1998), Sitri et Reboul (1998), Maingueneau (1999), Paveau (1999).

2. Avec l'énonciation, «on est passé d'une conception plurielle revêtant différents noms à un nom unique désignant une conception toujours plurielle » (S. Delasalle, citée par Puech, 1999: 56).

3. Colmez F. (dir) (2003), Français $3^{e}$, coll. Textes, Langages et Littératures, livre unique, Bordas (désigné infra par BOR-TLL) ; Fix-Combe N. (dir) (2003), Français 3e, séquences et expression, textes, images, pratique de la langue, Belin. (BEL) ; Pelet E., Haubert D., De Vulpillieres C., Voltz F., Paccalin M. (2003), Grammaire $3^{e}$, discours, textes, phrases, Belin (BEL-G); Potelet H. (dir) (2003), Français 3e, livre unique, Hatier (HAT) ; Morize-Toussain M., Coste H., Legendre-Torcolacci S., Morize G., Nguyen M., Raby V. (2003), Parcours méthodiques, 3e, Français, Hachette Education (H-EDUC); Carmignani F. (dir.), (2003), Français 3e, A travers mots, Bordas (BOR) ; Fize-Deneu P., Gapaillard C., Maubant Y., Français 3e, Didier (DID) ; Besson J.-J., Gallet S., Grivel S., Raymond M.-T. (2003), Français 3e, Texto collège, Hachette Education (HACH-TEX); Pages, A. (dir.), (2003), Français 3e, A mots ouverts, Nathan (NAT).

4. Nous utilisons ici le terme deixis au sens large, incluant, outre la deixis spatio-temporelle, la deixis personnelle.

5. Cette approche, soumise à peu de variation entre les manuels, donne lieu parfois à une ambigüité de la notion, interprétée soit comme un système de repérage formel, soit, dans un effort sans doute de simplification didactique, comme proche de la situation de communication. 
L'emploi de « contexte » cristallise cette ambigüité, observable dans l'extrait suivant : «Etudier l'énonciation, c'est reconstruire le contexte dans lequel un énoncé a été produit. Ainsi, la phrase : je veux que tu viennes tout de suite n'a de sens que si le lecteur sait qui sont je, tu, le lieu et qui désigne ici et le moment auquel correspond tout de suite. L'ensemble de ces informations constitue le contexte de l'énoncé, ce que l'on nomme la situation d'énonciation » (DID : 32).

6. Voir l'analyse critique des problèmes posés aux élèves par la reconnaissance des embrayeurs dans les énoncés dans l'article de D. Maingueneau, déjà cité, qui conclut en ces termes : «On imagine le dilemme dans lequel se trouvent les rédacteurs de manuels : ils ne peuvent donner des critères de reconnaissance valides, de peur d'être inintelligibles. Mais ils voudraient quand même produire des caractérisations qui aient quelque validité empirique. » (Maingueneau, 1999 : 26)

7. Le terme de déictique est souvent remplacé dans les manuels par celui d'indice d'énonciation.

8. Quand elles existent, elles se situent là encore au plan terminologique, par exemple en usant des termes " énonciateur » et « énoncé » : «on parle de modalisation quand l'énonciateur utilise dans son énoncé des procédés qui traduisent sa présence et son jugement ». (HAT: 326)

9. «Un énoncé comporte des marques plus ou moins visibles de la subjectivité de celui qui parle, c'est-à-dire des sentiments ou des jugements de valeurs, positifs ou négatifs, qu'il exprime » (NAT : 332).

10. Listes inspirées de la linguistique pragmatique et en particulier de l'ouvrage de référence de C. Kerbrat-Orecchioni (1980).

11. On peut supposer que l'élève n'aura pas de difficulté à utiliser « vert » ou « plat », mais aura peut-être plus de mal à mobiliser des marqueurs d'atténuation par exemple, tels que «probablement » ou des verbes épistémiques.

12. L'étymologie de «neutre » est de ce point de vue éclairante : neuter en latin renvoie à l'idée d'une absence de prise de position : ni l'un, ni l'autre. « Objectif » en revanche vient de objectivus/ objectum (objet). Dans le langage médical, les symptômes, signes objectifs, sont ceuxque le médecin peut constater (opposés à ceux, dits subjectifs, que seul le malade perçoit). Autrement dit, est objective toute personne dont les jugements ne sont altérés par aucune préférence d'ordre personnel.

13. Sur un terrain de football, un arbitre est précisément défini comme celui qui prend position (c'est là même le sens d'arbitrer ; un arbitre neutre serait un non sens !) et l'arbitre est d'autant mieux considéré qu'il sanctionne les joueurs avec le plus d'objectivité possible, c'est-à-dire de façon rationnelle, faisant appel à son jugement et non à ses affects.

14. Ainsi, dans un article de recherche, un énoncé caricatural tel que «Seule la linguistique de corpus appartient au champ de la linguistique», dépourvu a priori de "marques de subjectivité », nous semble bien plus "subjectif» (et donc peu crédible tel quel) que l'énoncé suivant, qui en est truffé : «on peut se demander dans quelle mesure la linguistique de corpus serait la seule à appartenir au champ de la linguistique ».

15. Cette conception est reprise à peu près dans les mêmes termes par tous les manuels.

16. La métaphore spatiale ne doit pas occulter le fait que l'on s'intéresse également ici à la deixis personnelle.

17. Cette articulation fait cependant l'objet de recherches actuelles. Dans la lignée des travaux de A. Rabatel (2004), F. Rinck, dans le cadre de sa thèse étudiant notamment les formes de « subjectivité représentée » dans l'article scientifique, insiste sur la nécessité de distinguer « une subjectivité déictique, qui concerne le sujet hic et nunc à l'origine du texte, et une subjectivité modale, en tant que repère des opérations modales " (Rinck, 2006).

18. Cette question, importante, du repérage dans l'espace énonciatif sera envisagée à travers ses implications pour la gestion de la textualité écrite.

19. Certains chercheurs utilisent responsabilité énonciative comme synonyme de prise en charge, mais il nous semble cependant que le terme de prise en charge témoigne davantage du 
caractère dynamique et processuel, responsabilité énonciative renvoyant à la simple assignation des sources.

20. Les documents d'accompagnement des programmes de 3e préconisent explicitement, dans un paragraphe intitulé «Ecrire pour autrui », des exercices mettant l'accent «sur la prise en charge par l'élève lui-même d'une opinion qu'il cherche à soutenir aux yeux de ses lecteurs en vue de la leur faire partager ", ou encore des exercices visant la prise de distance de l'élève par rapport à l'énoncé d'opinions divergentes ou conflictuelles.

21. Voir sur ce point les précisions fournies par Dendale et Coltier (2005).

22. Nous suivons en cela P. Dendale et D. Coltier (2005), dans leur article consacré à une discussion de la notion de prise en charge dans la théorie de la ScaPoLine.

23. Programme d'études du cours de français, Enseignement professionnel, Deuxième et troisième degrés, (2000:14-15).

\section{RÉSUMÉS}

Notre contribution porte sur la difficulté à didactiser une notion linguistique telle que l'énonciation dans les manuels. Comment présenter aux lecteurs potentiels (enseignants et élèves) un discours primaire intégrateur, autrement dit une forme de point de vue englobant, lorsqu'on a affaire à un domaine aussi mouvant que la linguistique énonciative ? L'observation critique de 9 manuels scolaires de français (pour la classe de $3^{\mathrm{e}}$ ), et plus particulièrement des chapitres dédiés à la modalisation et au discours rapporté, nous conduit à envisager la prise en charge énonciative comme entrée privilégiée dans le traitement didactique de l'énonciation.

Linguistic polyphony: modalities and reported speech in French textbooksOur study examines the difficulty of rendering both teachable and learnable the notions of linguistic polyphony (including modalities and reported speech) in school textbooks recently published in France. How is it possible to present a coherent point of view to teachers and to pupils, when one is dealing with such a rapidly evolving field as the linguistics of utterance construction? We analysed nine French textbooks intended for 13/14year old pupils, and in particular the chapters dealing with modalities and reported speech. The results lead us to argue that utterance endorsement (prise en charge énonciative) is the most suitable place at which to begin teaching the wider concept of linguistic polyphony.

\section{AUTEURS}

\section{FRANÇOISE BOCH}

Université Stendhal-Grenoble3, Lidilem

\section{FRANCIS GROSSMANN}

Université Stendhal-Grenoble3, Lidilem, E. A. 609 